\title{
détermination par fracturation hydraulique de l'état de contrainte à faible profondeur dans les roches élastiques et peu perméables
}

\author{
determination by hydraulic fracturing of the state of stress \\ at shallow depth in elastic and low permeable rocks
}

\section{Résumé}

\author{
Ph. CHARLEZ*
}

Cet article présente une nouvelle méthode de mesure de l'état de contrainte en surface en utilisant la fracturation hydraulique. Il est tout d'abord démontré que même en surface, il est possible d'obtenir des fractures verticales, ceci dépendant surtout du type d'obturateurs employés. Deuxièmement, partant des équations classiques de la fracturation hydraulique, on montre que seule la pression de fermeture est directement applicable en surface. De ce fait les trois inconnues que sont les deux contraintes horizontales et l'azimuth de l'une d'entre elles ne peuvent être évaluées qu'à partir de fractures d'orientations différentes. Deux méthodes sont alors présentées pour y arriver : soit l'utilisation de discontinuités naturelles, soit la modification de l'état de contrainte autour du puits à l'aide de verins courbes. Des essais effectués au fond d'une exploitation à ciel ouvert prouvent la validité de la méthode.

\section{Abstract}

This paper presents a new method of determining the state of stress at shallow depth. It is first shown that even at very low depth the fractures can be vertical, depending on the type of packer used. Secondly, a recall of the classical hydraulic fracturing shows that the only valid equation at shallow depth is the "instantaneous shut-in pressure". The three unknowns (two horizontal stresses and their azimuth) can be evaluated only if several different fracture orientations are created. Two methods are presented: either using of natural discontinuities or the modification of the state of stress around the wellbore with curved jacks. Experimental tests performed at the bottom of a quarry have proved the validity of the method.

- TOTAL-C.F.P. Centre de Recherches - Domaine de Beauplan, route de Versailles, 78470 Saint Rémy les Chevreuse. 


\section{INTRODUCTION}

Seul moyen d'investigation en profondeur $(1,2)$, la fracturation hydraulique n'est que rarement utilisée pour déterminer l'état de contrainte en surface. Elle est souvent supplantée par d'autres méthodes, surcarottage (3), vérin plat (4) dont la mise en œuvre est parfois compliquée et souvent coûteuse. La raison de ce choix a pour origine l'idée fausse que l'on se fait de la direction des fractures hydrauliques supposées perpendiculaires à la direction de la composante principale du tenseur contrainte. Une fracture hydraulique superficielle serait selon ce principe toujours horizontale et donc sans intérêt. Une analyse précise de l'état de contrainte autour du forage, montre qu'en réalité suivant le type d'obturateurs employés, les fractures peuvent être soit horizontales, soit verticales.

\section{DISCUSSION DE LA MÉTHODE CLASSIQUE DE MESURE}

La fracturation hydraulique consiste à isoler une portion de forage entre deux obturateurs étanches et à la mettre sous pression hydrostatique croissante jusqu'à ce qu'apparaisse pour une valeur particulière $\mathrm{P}_{\mathrm{R}}$ de la pression une fracture. Celle-ci est ensuite propagée sur une certaine distance (au moins trois à quatre fois le rayon du forage) par injection de fluide puis, à l'arrêt du pompage, le forage est maintenu sous pression. La pression chute alors brutalement, puis plus lentement et enfin tend à se stabiliser (voir fig. 1). Les courbes pression temps et la détermination de l'orientation de la fracture au niveau du forage peuvent être mises à profit pour déterminer certaines composantes du tenseur contrainte. Il nous paraît intéressant de discuter les équations qui permettent d'y arriver.

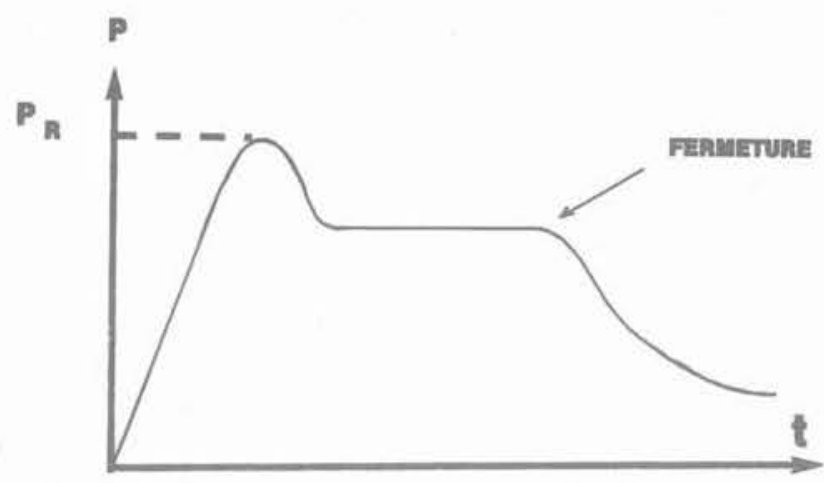

Fig. 1. - Diagramme de fracturation hydraulique. Fig. 1. - Classical diagram of hydraulic fracturing.

\section{1. État de contrainte à la paroi du forage} (fig. 2)

Dans le cas de roches sèches, l'état de contrainte à la paroi du forage, résulte de la superposition des trois chargements suivants :

2.1.1. L'état de contrainte à l'infini perturbé par la présence du forage sur une distance de trois à quatre fois son rayon. Si l'on suppose que la roche est linéairement élastique et isotrope, que la composante verticale du tenseur de contrainte $\sigma_{\mathrm{v}}$ est principale let par conséquent confondue avec l'axe $z$ lui aussi supposé vertical), on peut montrer qu'en coordonnées cylindriques, l'état de contrainte à la paroi d'un forage vertical dans un milieu infini, vaut (5) :

$$
\begin{gathered}
\sigma_{\beta \theta}=\left(\sigma_{H}+\sigma_{h}\right)-2\left(\sigma_{H}-\sigma_{h}\right) \cos 2(\beta-\xi) \\
\sigma_{z z}=\nu\left[\left(\sigma_{H}+\sigma_{h}\right)-2\left(\sigma_{H}-\sigma_{h}\right) \cos 2(\beta-\xi)\right]+\sigma_{v} \\
\sigma_{\phi \theta}=\sigma_{m z}=\sigma_{\theta z}=0
\end{gathered}
$$

$\sigma_{\mathrm{H}}$ et $\sigma_{\mathrm{h}}$ étant les contraintes horizontales principales à l'infini $\left(\sigma_{H}>\sigma_{h}\right)$. $\beta$ est compté positivement à partir du $\mathrm{N}$, dans le sens $\mathrm{N}-\mathrm{E}$ et $\xi$ est le plus petit angle de $\sigma_{\mathrm{H}}$ avec le Nord compté dans le même sens.

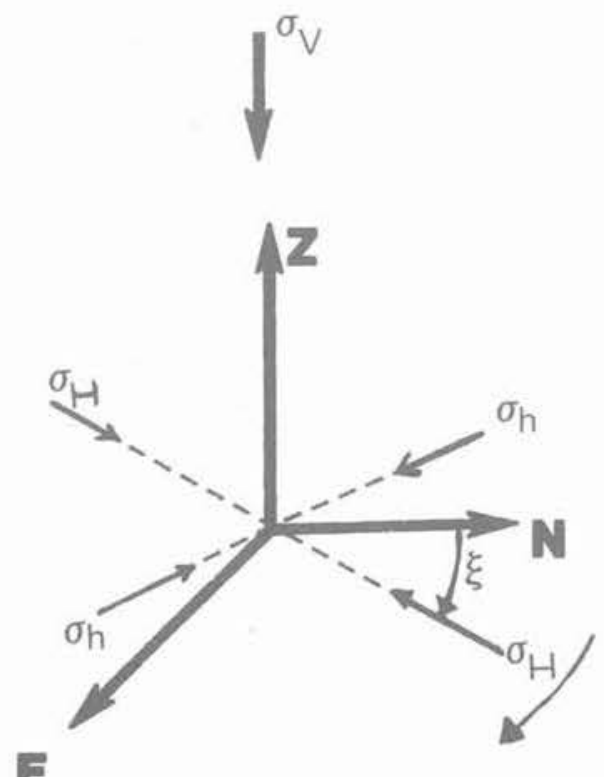

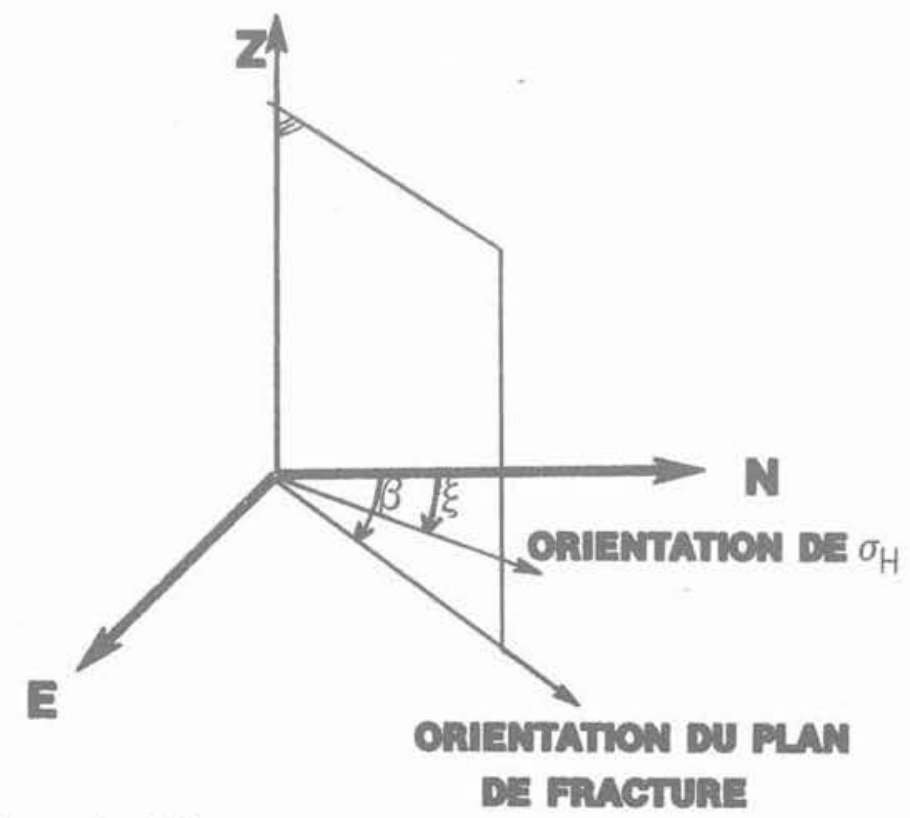

Fig. 2. - Axes de référence.

Fig. 2. - Reference axes. 
2.1.2. L'état de contrainte dû à la pression hydrostatique $\mathrm{P}$ appliquée à la paroi du forage entre les obsturateurs. Lorsque la distance entre ces obturateurs est suffisamment grande pour ne pas influencer l'état de contrainte dans la portion centrale de la région pressurisée, ce dernier vaut à la paroi (5).

$$
\begin{gathered}
\sigma_{p \theta}=\mathrm{P} \\
\sigma_{p \phi}=-\mathrm{P} \\
\sigma_{z z}=\sigma_{\rho \theta}=\sigma_{\rho z}=\sigma_{b 2}=0
\end{gathered}
$$

Dans le cas de roches saturées par un fluide sous pression, un terme correcteur doit être introduit dans les expressions (5) et (6). Lorsque la roche est peu perméable, ce terme se réduit à la valeur de la pression interstitielle. Dans le cas de mesures superficielles, il peut être négligé.

2.1.3. L'état de contrainte dû aux obturateurs. Lorsqu'ils sont scellés dans le forage, on peut considé. rer qu'ils y imposent un cisaillement uniforme dont la résultante est égale et opposée à la force engendrée par la pression sur les obturateurs (fig. 3). Kehle (6) a étudié ce problème tridimensionnel dans des hypothèses d'élasticité linéaire par la méthode des potentiels complexes. Il a montré (fig. 3) que pour ce type d'obturateur l'état de contrainte à̀ la paroi du forage au niveau de l'interface obturateur (cisaillement uniforme) chambre pressurisée (contrainte normale uniforme P) est tel que $\sigma_{z z}=-\mathrm{P}$ et $\sigma_{\theta 0}=0$.

Cette traction élevée parallèlement à l'axe du forage est due à la discontinuité du chargement et non à l'effort de cisaillement lui-même. C'est pourquoi, dans le cas d'obturateurs gonflables appliquant à la fois un cisaillement et une contrainte normale voisine de P, l'absence de discontinuité à l'interface chambre pressuriséeobturateur réduit la composante $\sigma_{z}$ à environ $10 \%$ de la pression appliquée dans le forage.

Le champ de contrainte total à la rupture (soit pour la valeur $\mathrm{P}_{\mathrm{R}}$ de la pression $\mathrm{P}$ ), résulte de la superposition des trois composantes élémentaires soit,

dans la chambre pressurisée

$\sigma_{\theta}=\left(\sigma_{H}+\sigma_{h}\right)-2\left(\sigma_{H}-\sigma_{h}\right) \cos 2(\beta-\xi)-P_{R}$ (8) et au niveau du passage chambre hydrauliqueobturateur,

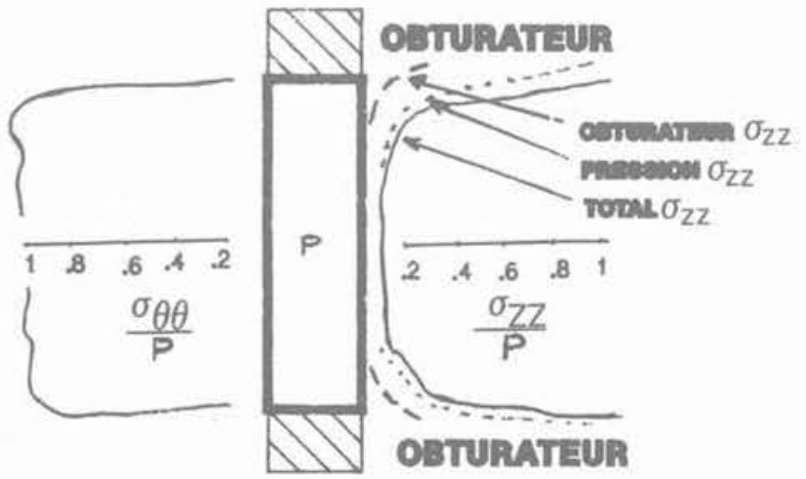

Fig. 3. - Influence des obturateurs. Fig. 3. - Packers influence. $\sigma_{z z}^{(1)}=v\left[\sigma_{\mathrm{H}}+\sigma_{\mathrm{h}}-2\left(\sigma_{\mathrm{H}}-\sigma_{\mathrm{h}}\right) \cos 2(\beta-\xi)\right]+\sigma_{\mathrm{v}}-\mathrm{P}_{\mathrm{R}}$

dans le cas d'obturateurs scellés et

$$
\sigma^{(2)}=v\left[\sigma_{H}+\sigma_{h}-2\left(\sigma_{H}-\sigma_{h}\right)\right.
$$

$\cos 2(\beta-\xi)]+\sigma_{y}-0,1 \mathrm{P}_{\mathrm{B}}$

dans le cas d'obturateurs gonflables.

Pour savoir si la fracture créée sera horizontale ou verticale, il faut comparer les pressions $\mathrm{P}_{\mathrm{R} \theta}$ et $\mathrm{P}_{\mathrm{RZ}}$ pour lesquelles $\sigma_{6 \beta}$ et $\sigma^{(1)}(2)$ atteignent la résistance à la traction du matériaux $R_{T}$. La fracture sera donc verticale si :

$$
\mathrm{P}_{\mathrm{R} \theta}<\mathrm{P}_{\mathrm{R} 2}^{(1)(2)}
$$

(10 bis)

Dans le cas d'une roche isotrope (du point de vue de sa résistance à la traction $R_{T}$ ), une fracture verticale s'amorçant nécessairement dans la direction de $\sigma_{H}$ $(\beta=\xi)$, dans le cas d'obturateurs scellés la fracture sera verticale si :

$$
\sigma_{\mathrm{v}}>(1-u)\left(3 \sigma_{\mathrm{h}}-\sigma_{\mathrm{H}}\right)
$$

Alors que dans le cas d'obturateurs gonflables

$$
\sigma_{v}>(0,1-v)\left(3 \sigma_{h}-\sigma_{H}\right)
$$

les chances d'obtenir une fracture verticale augmentent donc avec la profondeur et avec l'intensité du déviateur. Dans le cas d'obturateurs gonflables ( $\nu>0,1)$ la fracture sera toujours verticale et ce quelle que soit la profondeur.

Si le milieu est anisotrope (c'est-à-dire fracturé naturellement) avec une résistance horizontale $R_{H}^{\top}$ et une résistance verticale $R_{v}^{\mathrm{T}}(\beta)$ variant avec l'azimuth, les relations (11) et (12) deviennent :

- Dans le cas d'obturateurs scellés :

$\sigma_{v}>(1-\nu)\left[\left(\sigma_{\mathrm{H}}+\sigma_{h}\right)-2\left(\sigma_{\mathrm{H}}-\sigma_{h}\right) \cos 2(\beta-\xi)\right]$

$+\mathrm{R}_{\mathrm{v}}^{\mathrm{T}}(\beta)-\mathrm{R}_{\mathrm{H}}^{\mathrm{T}}$

- Dans le cas d'obturateurs gonflables :

$\sigma_{v}>(0,1-\nu)\left[\left(\sigma_{H}+\sigma_{h}\right)-2\left(\sigma_{H}-\sigma_{h}\right) \cos 2(\beta-\xi)\right]$

$+0,1 R_{v}^{\top}(\beta)-R_{H}^{\top}$

Dans ce dernier cas, seule une forte anisotropie horizontale (schistosité par exemple) peut donner lieu à une fracturation horizontale.

En conclusion, lemploi d'obturateurs gonflables permet d'obtenir en surface une fracture verticale dans presque tous les cas.

\subsection{Les équations classiques de la fracturation hydraulique}

Lorsque les fractures sont verticales, les valeurs de $\sigma_{h}$, $\sigma_{\mathrm{H}}$ et $\xi$ peuvent être déterminées à l'aide des équations décrites ci-après.

L'équation de rupture suppose que la fracture s'amorce dans la direction $\beta$ où la contrainte $\sigma_{\rho \%}$ à la périphérie du forage atteint la première la valeur locale de la résistance à la traction de la roche $\mathrm{R}$ t. Dans le cas où $R$ : est isotrope (c'est-à-dire indépendante de $\beta$ ) la fracture est alignée sur $\sigma_{\mathrm{H}}$. Dans le cas général, on peut écrire :

$\mathrm{R}_{i}(\beta)=\left(\sigma_{\mathrm{H}}+\sigma_{\mathrm{h}}\right)-2\left(\sigma_{\mathrm{H}}-\sigma_{\mathrm{h}}\right) \cos 2(\beta-\xi)-\mathrm{P}_{\mathrm{R}}(15)$ L'expérience montre (2) que cette équation est très imprécise de par la nature aléatoire de $\mathrm{R}_{\mathrm{t}}(\beta)$; la précision que l'on peut attendre de cette mesure est souvent inférieure à l'ordre de grandeur des déviateurs $\sigma_{H}-\sigma_{h}$ auxquels on peut s'attendre en surface. Cette équation est pour cette raison difficilement utilisable. Pour pallier à cette difficulté, certains auteurs (7) ont proposé d'utiliser la pression de réouverture c'est-à-dire la pression 

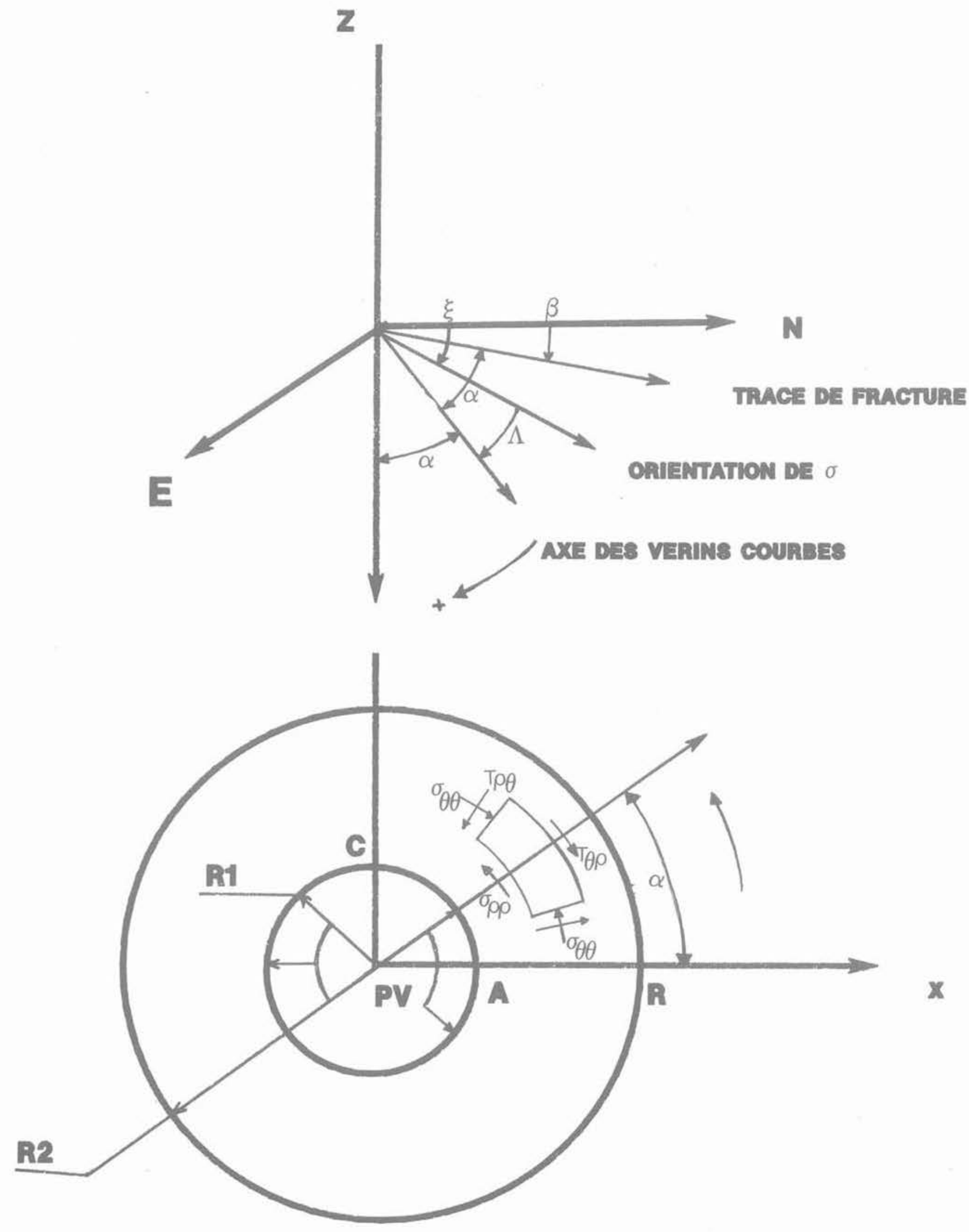

Fig. 4. - Problème des vérins courbes.

Fig. 4. - The curved jacks problem. 
requise pour réouvrir une fracture hydraulique déjầ développée après que la pression interstitielle fut retournée à sa valeur initiale dans tout le massif. L'équation correspondant à cette opération est identique à l'équation (15) avec $\mathrm{R}_{1}(\beta)=0$, la réouverture ne demandant aucune énergie de séparation. Cette équation est rarement utilisée pour deux raisons. D'une part elle dépend fortement de la dunamique de l'écoulement mais surtout, elle est inutilisable lorsque la fracture est déjà ouverte au niveau du forage c'est-à-dire lorsque $\sigma_{\text {pf }}<0$ avant toute pressurisation, ce qui s'écrit :

$$
\sigma_{\mathrm{h}}<\sigma_{H} \frac{2 \cos 2(\beta-\xi)-1}{2 \cos 2(\beta-\xi)+1}
$$

La troisième méthode utilise la relation entre les composantes horizontales du tenseur géostatique à la contrainte normale $\sigma_{n}$ s'exerçant sur le plan de la fracture. C'est la seule qui puisse être utilisée sans ambiguité. En effet, on peut montrer que la libération du cisaillement préexistant (nul si la fracture est parallèle à $\sigma_{H}$ ) n'affecte en rien $\sigma_{n}$. qui après fracturation reste égale à la projection du champ de contrainte $z$ sur la direction de la normale $\vec{n}$ au plan de la fracture soit :

$$
\sigma_{\mathrm{n}}=[\mathrm{g} \overrightarrow{\mathrm{n}}] \overrightarrow{\mathrm{n}}
$$

En surface $(z<3 \mathrm{~m}) \sigma_{\mathrm{v}}$ pouvant être négligé, cette formule peut être explicitée linéairement en posant

$$
\begin{gathered}
X=\sigma_{H}+\sigma_{h} \\
Y=\left(\sigma_{H}-\sigma_{h}\right) \cos 2 \xi \\
Z=\left(\sigma_{H}-\sigma_{h}\right) \sin 2 \xi
\end{gathered}
$$

sous la forme

$$
X+Y \cos 2 \eta+Z \sin 2 \eta=4 \sigma_{n} /(1-\cos 2 \phi)
$$

où $\eta=\beta+\left(\frac{\pi}{2}\right)$ et $\Phi$ sont respectivement les orientations de cette normale par rapport au nord et la verticale. Au même titre que $\sigma_{H} \sigma_{h}$ et $\xi, X, Y$ et $Z$ varient avec la profondeur.

La mesure de $\sigma_{n}$ peut s'effectuer de deux façons, en considérant la pression de fermeture instantanée et la pression de propagation à faible débit.

En effet, la pression requise pour propager une fracture peut être décomposée comme suit

$$
\mathrm{P}=\sigma_{n}+\delta \sigma_{n}+\mathrm{P}_{\mathrm{C}}+\mathrm{P}_{\mathrm{k}}
$$

où :

$\sigma_{n}$ est la contrainte normale à la fracture.

$\delta \sigma_{n}$ l'incrément supplémentaire de pression pour que la fracture s'ouvre,

$\mathrm{P}_{C}$ la perte de charge dans la fracture,

$P_{K}$ l'effort de cohésion qu'il faut vaincre pour créer un élément de fracture supplémentaire.

Pour des fractures à propagation radiale (c'est-à-dire non confinées entre deux épontes), $\mathrm{P}_{\mathrm{K}}$ diminue avec l'extension et devient rapidement négligeable dès que la fracture atteint des dimensions métriques. Si en plus, le débit d'injection est faible (quelques litres par minute), ce qui ne sera possible que pour des roches peu perméables, la perte de charge est elle aussi négligeable et, dans ce cas la pression de propagation est légèrement supérieure à la contrainte $\sigma_{n}$ normale à la fracture.

$\sigma_{n}$ peut aussi s'évaluer à l'aide de la pression de fermeture instantanée ISIP ${ }^{*}$ dont nous explicitons ci-dessous

- ISIP : Instantaneous Shut in Pressure. la signification. Une fois que le pompage est arrêté, la frature est maintenue sous pression. On assiste alors à une décroissance de la pression et à la fermeture progressive de la fracture (fig. 1). Deux raisons expliquent cette décroissance de la pression : tout d'abord la propagation apost arrêt de pompage * dont résulte une augmentation rapide du volume de la fracture, donc une chute de pression pratiquement instantanée. aucun apport de fluide supplémentaire n'étant assuré. Lorsque les conditions de propagation en tête de fracture ne seront plus requises, seules les fuites par perméabilité à travers les faces de la fracture peuvent expliquer une chute de pression supplémentaire. Le changement de pente observé sur la courbe de fermeture correspond à l'arrêt de la propagation. La valeur de la pression qui y correspond est assez proche de la valeur de $\sigma_{n}$. C'est ce que l'on appelle l'ISIP. L'ISIP surestime toutefois $\sigma_{n}$ puisque la fracture est à ce stade toujours ouverte. Il y a donc en réalité trois configurations différentes au cours de la fermeture : la propagation post «arrêt de pompage *, la fracture qui se referme par percolation du fluide uniquement, la fracture fermée. La valeur de $\sigma_{n}$ correspond en réalité à la transition entre les deux dernières configurations. Toutefois, cette transition n'est pas souvent observable au contraire de la première (correspondant au changement de pente mentionné plus haut). Ce dernier est d'autant plus facile à estimer que la roche est peu perméable puisque dans le cas contraire la chute de pression due aux fuites vient masquer l'arrêt de la propagation. Nous nous contenterons d'évaluer $\sigma_{n}$ au changement de pente. Plusieurs types de constructions géométriques permettent de l'estimer (8). Ces techniques sont toutefois loin de faire toujours l'unanimité, car aucune base théorique n'est réellement établie.

Le calcul des trois inconnues $\sigma_{H}, \sigma_{h}$ et है (c'est-à-dire X. $\mathrm{Y}, \mathrm{Z})$ n'est donc pas possible à partir de la méthode classique (équations de rupture, réouverture, fermeture) puisqu'en surface il a été montré que seule l'équation (19) était utilisable.

Dès lors s'il s'avérait possible de développer des fractures hydrauliques dans différentes directions indépendamment de l'état de contrainte régional, ces fractures pourraient être utilisées comme de véritables vérins plats et permettraient la mesure de la contrainte normale supportée par des plans d'orientations variées. On aboutirait alors à un système d'équations linéaires (du type 19) à trois inconnues X, Y, Z. La suite de larticle sera centrée sur la possibilité d'appliquer cette méthode de mesure de contraintes exclusivement à l'aide de la mesure de $\sigma_{n}$ le long de fractures d'orientations différentes et donc sur les possibilités effectives de les créer à faible profondeur.

\section{AMORCE ET PROPAGATION D'UNE FRACTURE HYDRAULIQUE ORIENTÉE}

Par fracture orientée, nous faisons référence à des fractures hydrauliques dont l'orientation n'est pas perpendiculaire à la direction de la composante principale minimum du tenseur de contrainte.

Deux méthodes peuvent être envisagées. 


\subsection{Fracturation hydraulique orientée naturelle}

Dans le cas de roches isotropes et selon la théorie classique, les fractures sont parallèles à la contrainte principale maximum horizontale. Si par contre le massif présente une anisotropie de résistance ayant pour origine une fissuration naturelle (failles, schistosité, joints de calcite), la rupture ne surviendra pas nécessairement parallèlement à $\sigma_{\mathrm{H}}$ mais dans une direction où la contrainte à la paroi du forage atteindra la première la résistance locale soit :

$$
\sigma_{\infty \theta}(\beta)=\mathrm{R}_{1}(\beta)
$$

Dans le cas où la discontinuité n'est pas recimentée, $R_{t}(\beta)$ sera par ailleurs nulle.

Lorsque le plan de fracture est incliné par rapport aux directions des contraintes principales régionales horizontales, il y a relâchement de la composante de cisaillement dans ce plan. De ce fait, lors de la fermeture de la fracture les lèvres ne reviennent pas parfaitement en contact. Toutefois, ce relâchement ne modifie nullement la composante normale de la contrainte supportée par ce plan qui reste après fracturation égale à celle qui préexistait à la fracturation, comme nous l'avons déjà précisé (voir 2.2.).

\subsection{Fracturation hydraulique orientée artificielle}

La méthode consiste à modifier l'état de contrainte à la périphérie du trou en superposant à la pression hydrostatique $\mathrm{P}_{\mathrm{H}}$ une pression sectorielle symétrique $\mathrm{P}_{\mathrm{v}}$ appliquée uniformément à l'aide de vérins courbes sur deux secteurs de portance angulaire $2 \alpha$. La résolution du problème des vérins courbes (fig. 4) peut être abordée par la théorie de l'élasticité $(5,9)$ à l'aide de la théorie des potentiels complexes soit pour un cylindre creux infiniment long soit pour un massif infini percé d'un trou (voir l'annexe).

A la paroi du forage, l'expression de la contrainte tangentielle tend vers une solution exacte, discontinue lorsqu'on passe de la zone non chargée à la zone chargée. En particulier, la contrainte tangentielle $\sigma_{\theta \theta}$ due aux seuls vérins est telle que :

$\left.\sigma_{\theta \theta}=-\left(4 \mathrm{P}_{v} \alpha\right) / \pi\right)+\mathrm{P}_{y}$

dans la zone chargée et

dans la zone non chargée.

La contrainte totale due à la superposition des états de contrainte résultant des pressions hydrostatiques et sectorielles ainsi que du champ de contrainte géostatique vaudra à la paroi :

$$
\begin{gathered}
\left(\sigma_{\theta \theta}\right) 1=\left(\sigma_{H}+\sigma_{h}\right)-2\left(\sigma_{H}-\sigma_{h}\right) \cos 2(\beta-\xi) \\
-\left(4 \mathrm{P}_{v} \alpha / \pi\right)+\mathrm{P}_{v}-\mathrm{P}_{H}
\end{gathered}
$$

dans la zone chargée par les vérins, et :

$$
\begin{gathered}
\left(\sigma_{\theta e}\right) 1=\left(\sigma_{H}+\sigma_{h}\right)-2\left(\sigma_{H}-\sigma_{h}\right) \cos 2(\beta-\xi) \\
-\left(4 \mathrm{P}_{v} \alpha / \pi\right)-\mathrm{P}_{\mathrm{H}}
\end{gathered}
$$

dans la zone non chargée.

Si la direction de $\sigma_{H}$ se trouve dans le secteur non chargé par les vérins courbes, leur effet est de diminuer la valeur de la pression hydrostatique requise pour amorcer la fracture hydraulique (de $\left.4 \mathrm{P}_{\mathrm{v}} / \pi\right)$ mais la direction reste inchangée (c'est-à-dire parallèle à $\sigma_{H}$ ).
Par contre si la direction de $\sigma_{\mathrm{H}}$ se trouve dans le secteur d'action des vérins courbes, alors la fracture peut s'amorcer parallèlement à la directrice limite du secteur chargé la plus proche de $\sigma_{H}$ (c'est-à-dire pour un angle $\beta=\xi+\Lambda-\alpha, \Lambda$ définissant l'orientation de l'axe des vérins par rapport à $\sigma_{H}$ ). La pression $\mathrm{P}_{\mathrm{v}}$ dans les vérins courbes devra toutefois être suffisante pour que (les compressions sont supposées positives) :

$\mathrm{P}_{\mathrm{H}}$ rupture $(\beta=\xi)>\mathrm{P}_{\mathrm{H}}$ rupture $(\beta=\xi+\Lambda-\alpha)(26)$ sans quoi la rupture s'opèrera parallèlement à $\sigma_{H}$ soit si :

$$
P_{v}>2\left(\sigma_{H}-\sigma_{b}\right)[1-\cos 2(\Lambda-\alpha)]
$$

Dans le cas de la cellule développée à l'Institut de Physique du globe de Paris, que nous avons utilisée $\alpha=\pi / 4$. La condition s'écrira :

$$
P_{v}>\alpha\left(\sigma_{H}-\sigma_{h}\right)(1-\sin 2 \Lambda)
$$

La pression requise pour que la fracture soit orientée autrement que suivant $\sigma_{H}$ est donc proportionnelle au déviateur et diminue si $\Lambda$ augmente. Si $\Lambda=\alpha$ le bord du vérin se trouve dans la direction de $\sigma_{H}$ et la fracture s'amorcera dans cette direction.

Une fois amorcée, la fracture orientée doit être propagée sur une distance suffisante dans sa propre direction afin de s'affranchir de la concentration de contrainte due à la présence du forage.

Toutefois, le calcul montre qu'une amorce orientée propagée de façon quasi statique par une pression hydraulique tourne instantanément et se réaligne sur $\sigma_{\mathrm{H}}$, phénomène d'autant plus marqué pour des fractures orientées à $45^{\circ}$ par rapport aux contraintes. Toutefois, lamorce n'est jamais quasi statique et, au départ le taux de restitution d'énergie excède largement l'énergie de surface. L'amorce est toujours suffisamment longue pour que la mesure soit possible. Les essais décrits dans la suite viendront le confirmer.

\section{LA CELLULE IPG}

(Institut de Physique du Globe de Paris)

La cellule IPG est une sonde de $72 \mathrm{~mm}$ de diamètre couplant sur un même appareil les deux systèmes de pressurisation proposés (pressions hydrostatique et sectorielle) (fig. 5)

Elle se compose essentiellement d'une partie centrale (destinée à l'injection du fluide et à l'ancrage des vérins), de deux obturateurs, gonflables et autoserrables, et de deux vérins courbes de portance $90^{\circ}$ chacun.

Les vérins sont déformables; ils se présentent sous la forme d'un double feuillet métallique de $1 \mathrm{~mm}$ d'épaisseur. Ils résultent de l'emboutissage de tubes inox dans une matrice prévue à cet effet.

L'appareil a d'abord été testé dans un tube d'acier de $1 \mathrm{~cm}$ d'épaisseur dont la déformation a été évaluée à l'aide de jauges résistives disposées radialement sur la face externe du tube. Les valeurs mesurées dans différents azimuths par rapport à l'axe des vérins $(0, \pi / 2, \pi$, $\pi / 2$ ) ont alors été comparées aux valeurs prévues par la théorie de l'élasticité.

Sur les courbes de la figure 6 apparaissent à la fois l'anisotropie symétrique du déplacement au contour ainsi 


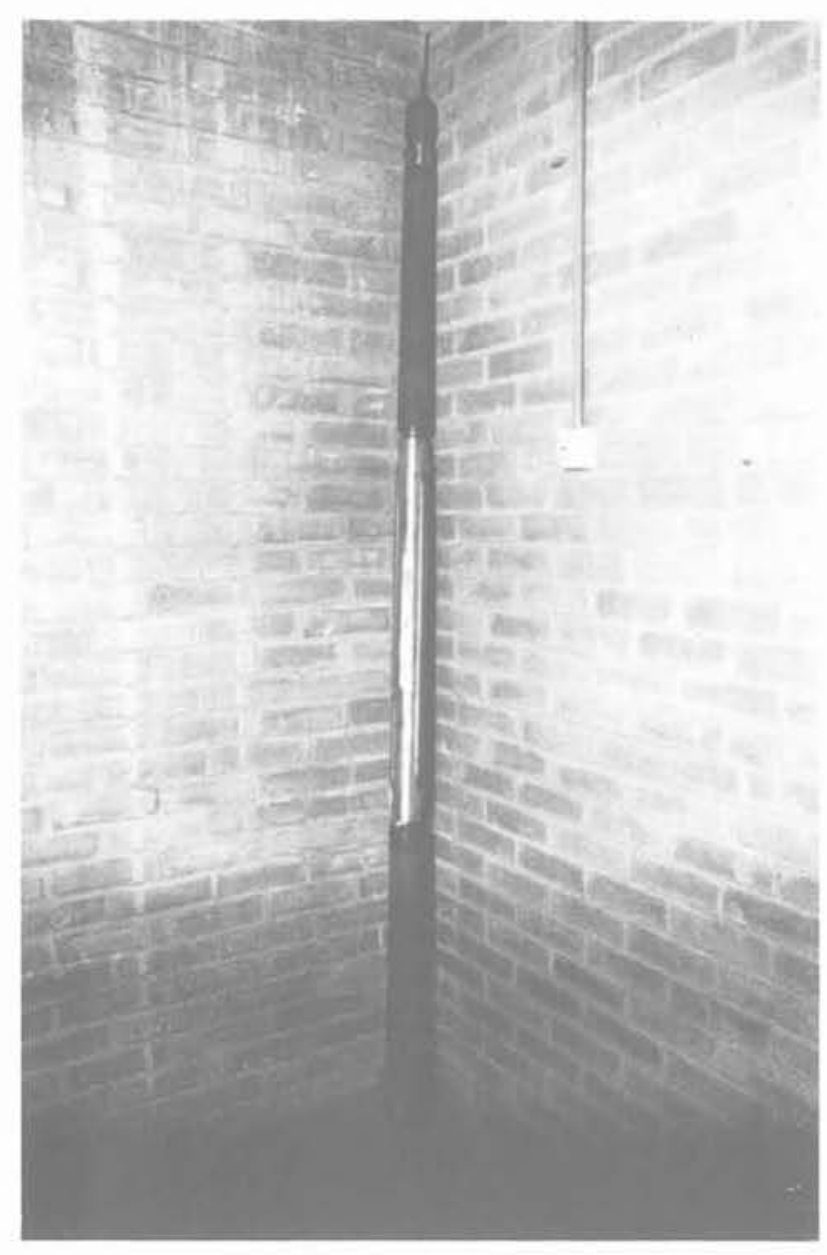

Fig. 5. - Cellule IPG.

Fig. 5. - IPG cell.

que la réponse des vérins qui jusqu'à 1 MPA environ se déforment sans déformer le tube.

La comparaison est assez satisfaisante :

\begin{tabular}{|c|c|c|}
\hline$\theta$ & $\begin{array}{c}\epsilon \text { mesuré } \\
\left(10^{-6} / \text { bar }\right)\end{array}$ & $\begin{array}{c}\epsilon \text { calculé } \\
\left(10^{-6} / \text { bar }\right)\end{array}$ \\
\hline $\begin{array}{c}0 \text { ou } \pi \\
\pi / 2 \text { ou } 3 \pi / 2\end{array}$ & $\begin{array}{c}-10,81 \\
+7,59\end{array}$ & $\begin{array}{c}-9,45 \\
+7,85\end{array}$ \\
\hline
\end{tabular}

L'écart compris entre 5 et $10 \%$ montre que les vérins ne sont pas efficaces sur toute leur portance.

\section{RÉSULTATS EXPÉRIMENTAUX}

Une série d'essais a été réalisée au fond d'une exploitation à ciel ouvert située dans le Sud de la Belgique (Soignies). La roche, connue sous le nom local de "petit granite», est un calcaire crinoidique très compact, très raide et fortement imperméable (porosité inférieure au millième, module d'élasticité supérieur à $50 \mathrm{GPa}$ et perméabilité inférieure au microdarcy).
Trois forages voisins superficiels ( $A, B$ et $C$ ), préalablement carottés ont permis d'effectuer un certain nombre d'essais dont six ont pu être retenus (fig. 7). Un seul (essai 11) a été effectué à l'aide des vérins courbes.

La contrainte normale à la fracture a été évaluée à la fois à l'aide de la pression de fermeture instantanée et dans certains cas avec la pression de propagation à faible débit.

L'orientation $\eta$ du plan de la fracture $(\eta=\beta+(\pi / 2))$ ainsi que l'inclinaison $\phi$ de la normale du plan de la fracture par rapport à la verticale ont pu être mesurées grâce à un packer d'impression. Pour chacune des mesures de $\sigma_{n}, \eta$ et $\phi$, des marges d'erreur ont étéappréciées. La bonne concordance entre la pression de fermeture instantanée et la pression de propagation à faible débit a notamment été prise en considération pour évaluer la précision sur $\sigma_{n}$.

Les profondeurs indiquées correspondent à la transition "obturateur supérieur, chambre hydraulique». Ces profondeurs n'ont d'ailleurs qu'une importance toute relative puisque la contrainte verticale est négligée dans le modèle.

En remplaçant les valeurs ci-dessus dans l'équation (17) on obtient un système linéaire surdéterminé de six équations à trois inconnues.

Nous avons alors introduit les valeurs ci-dessus dans un programme d'inversion généralisé disponible à l'IPG. Les résultats sont les suivants :

$$
\begin{gathered}
\sigma_{\mathrm{H}}=32 \pm 2 \mathrm{bar} \\
\sigma_{\mathrm{h}}=6.5 \pm 1.5 \mathrm{bar} \\
\xi=\mathrm{N} 82^{\circ} \mathrm{E} \pm 2.5^{\circ}
\end{gathered}
$$

Le champ de contrainte est donc fortement anisotrope $\left(\sigma_{H}=5 \sigma_{h}\right)$ et la valeur des contraintes faible. Ces valeurs justifient donc toutes les remarques développées à propos de l'utilisation des pressions de rupture et de réouverture.

Trois orientations franchement différentes ont été observées. Une seule fracture est pseudoparallèle à $\sigma_{H}$ (c'est-à-dire pseudoparallèle à la paroi abrupte de l'excavation (fig. 7). Quatre fractures se sont développées dans des discontinuités naturelles (essais $3,6,7$ et 8) celles-ci étant pseudoparallèles à la faille principale traversant la carrière. Pour l'essai $n^{\circ} 11$, le seul effectué à l'aide des vérins courbes (dont l'axe avait été placé E-W), la fracture s'est amorcée en formant un angle de $39^{\circ}$ avec leur axe. Un surcarottage postérieur a montré que la fracture s'était propagée dans sa propre direction sur $15 \mathrm{~cm}$ après quoi elle s'est réalignée sur $\sigma_{\mathrm{H}}$. Cette longueur est donc suffisante pour s'affranchir de la concentration de contrainte due au forage ( $7 \mathrm{~cm}$ de diamètre). Il faut toutefois limiter au minimum les volumes injectés si l'on veut que la première mesure de pression de fermeture corresponde à la contrainte supportée par le plan orienté.

Quoi qu'il en soit, on observe donc bien des fractures verticales ou pseudo-verticales et non horizontales (la fracture ayant un pendage de $12.5^{\circ}$ correspond à l'inclinaison du joint de calcite dans lequel elle s'est amorcée).

\section{CONCLUSIONS}

On peut déduire de cette étude les conclusions suivantes: 
Fig. 6. - Etalonnage des vérins courbes en laboratoire.

Fig. 6. - Calibration of curved jacks.
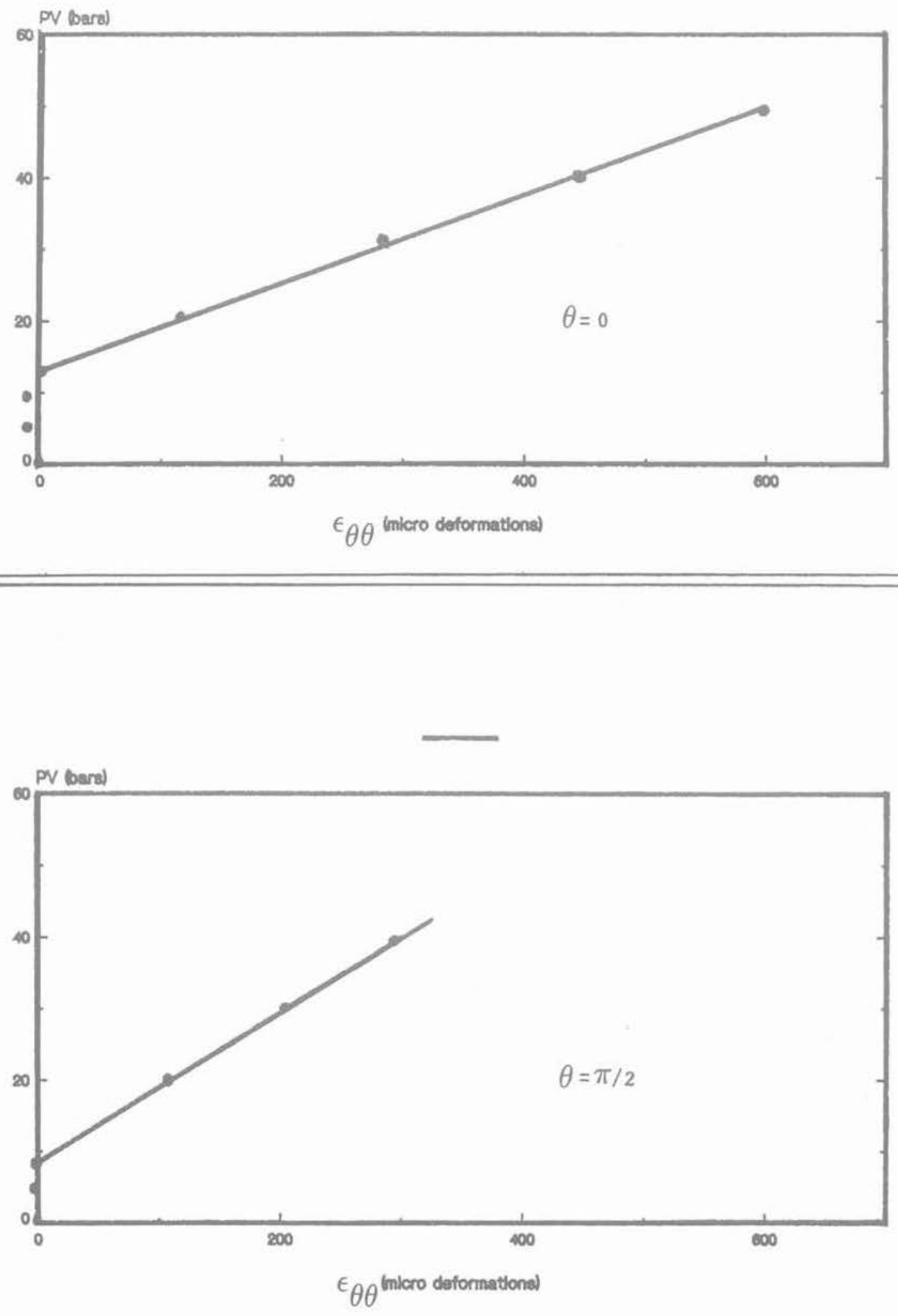

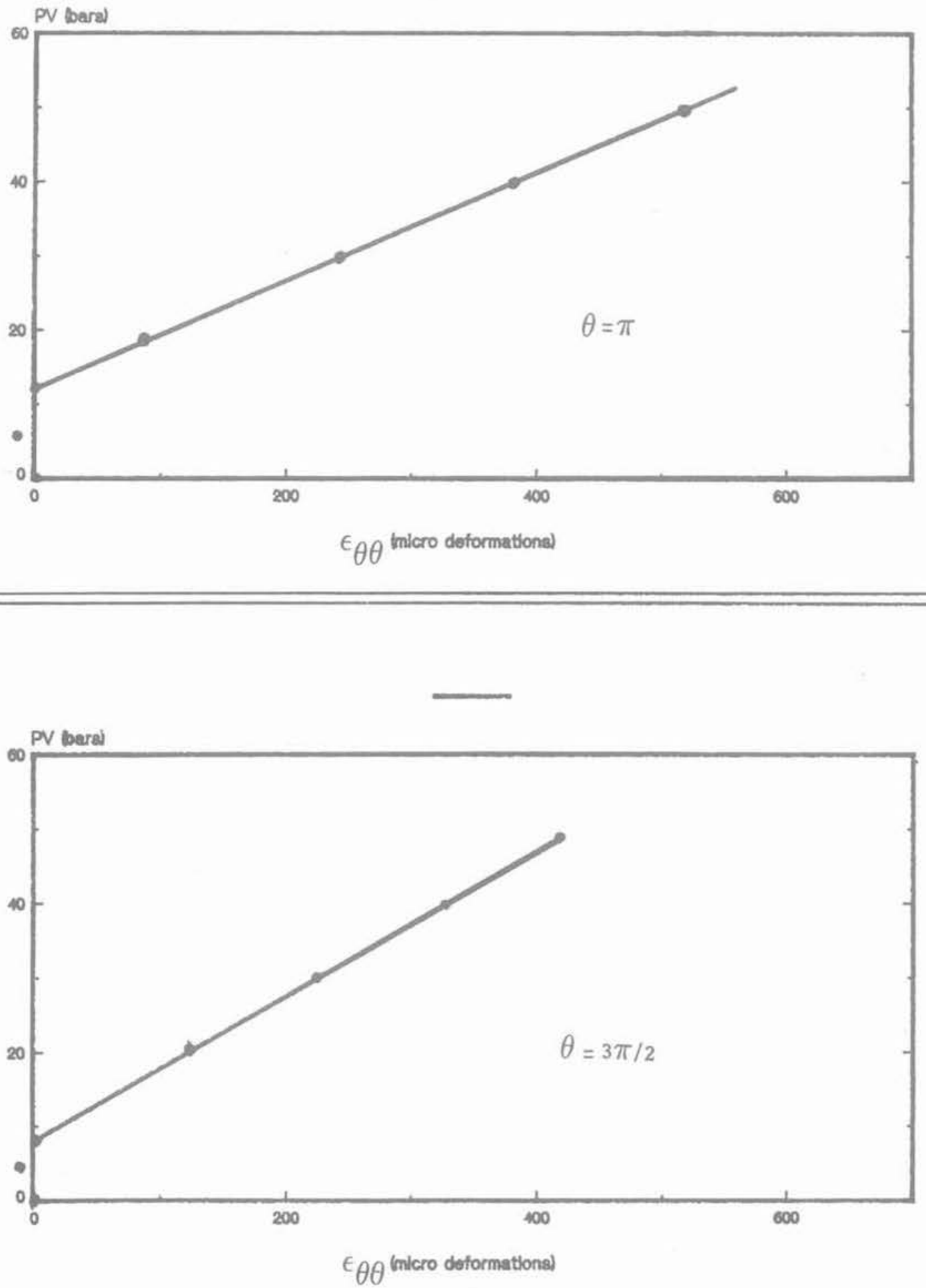

Fig. 6. - Etalonnage des vérins courbes en laboratoire.

Fig. 6. - Calibration of curved jacks. 
FALAISE

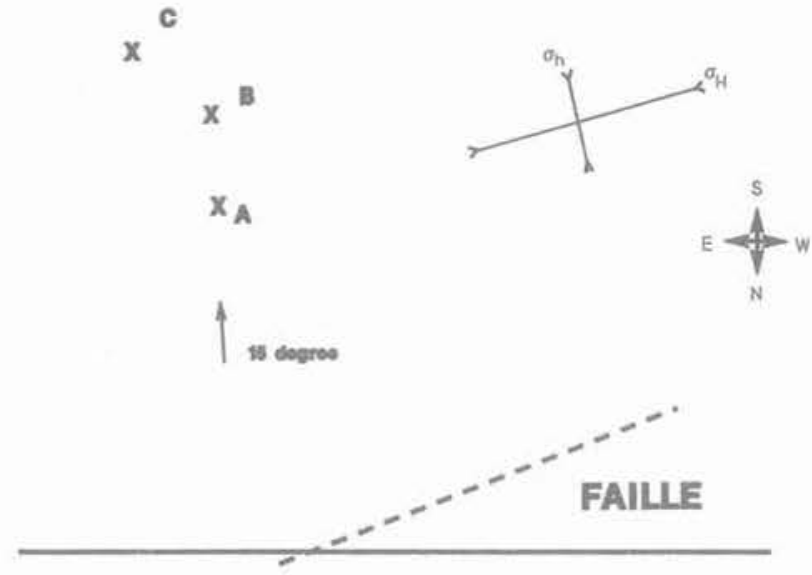
Fig. 7. - Plan de la carrière et orientation
du champ de contrainte superficiel.

Fig. 7. - Orientation of the state of stress at the bottom of the quarry.

- Du fait du type de chargement (obturateurs gonflables) et conformément à la théorie de Kehle, les fractures bien que superficielles sont verticales. Ceci justifie donc bien l'emploi d'obturateurs gonflables.

- Dans le cas traité le rapport $\sigma_{\mathrm{H}} / \sigma_{\mathrm{h}}$ étant supérieur à 3 , et, la valeur absolue des contraintes d'un ordre de grandeur comparable à l'incertitude sur la résistance à la traction de la roche, les équations de rupture et de réouverture ne sont pas utilisables. La mesure de la seule contrainte normale au plan de fracture (par la pression de fermeture instantanée et la pression de propagation à faible débit) nécessite de multiplier le nombre d'orientations (au moins trois) et de prolonger les fractures dans leur propre direction sur des distances suffisantes. L'emploi de vérins courbes résoud artificiellement ce problème.

- Enfin, elle confirme le rôle essentiel de l'anisotropie de résistance sur l'amorçage des fractures hydrauliques en surface. L'hypothèse classique qu'une fracture est toujours parallèle à $\sigma_{\mathrm{H}}$ est dans ce cas presque toujours erronée. L'azimuth de $\sigma_{H}$ doit donc être calculé non mesuré.

\section{REMERCIEMENTS}

Ce travail a été réalisé à l'Institut de Physique de Globe de Paris sous la direction de F.H. CORNET. Il a été financé par la Commission des Communautés Européennes et la Compagnie Française des Pétroles.

\section{BIBLIOGRAPHIE}

1. ROEGIERS J.C. (1975). The development and evaluation of a field method for in situ stress determination using hydraulic fracturing. Final report Departement of Army Contract N DACW 45-74 $-\mathrm{C}-006$.

2. HAIMSON B., FAIRHURST C. (1969). In situ stress determination at great depth by means of hydraulic fracturing. 11th Symposium on rock mechanics, June 1969, Berkeley, pp. 559-584.

3. BONNECHERE F.J. (1970). Contribution à la détermination de l'état de contrainte dans les mas. sifs rocheux. Thèse de doctorat en sciences appliquées, Université de Liège (Belgique).

4. TINCELIN E. (1951). Les études de pressions de terrain entreprises dans les mines de fer de Lor. raine. Conférence sur les pressions de terrain, 24-28 avril 1951.

5. JAEGER J.C. ET COOK N.W.G. (1969). Fundamental of Rock mechanics. Methuen and Co Ltd.

6. KEHLE R.D. (1964). Determination of Tectonic stresses through analysis of hydraulic well fracturing. JGR, vol. $69, \mathrm{n}^{\circ} 2$, pp. 259-273, 3 fig.

7. ZOBACK M., POLLARD D. (1978). Hydraulic fracturing propagation and the interpretation of pressure-time records for in situ stress determinations. 19th US Symposium on rock mechanics, Stateline, Nevada.

8. McLENNAN, ROEGIERS J.C. (1982), How instantaneous are ISIP? SPE 11064.

9. MUSKHELISVILI N.I. (1953). Some basic problems of the mathematical theory of Elasticity. $3^{e}$ Revised Edition and augmented edition; traduit du russe par JRM. Radock; Nordhof-Ltd Gronigen, Holland.

10. CORNET F.H. (1981). Etude sur la propagation des fractures hydrauliques dans les roches. Rapport pour la C.E.E. - Référence EUR 7287, MF 80 pages.

11. CHARLEZ Ph. (1984). Détermination de l'état de contrainte dans les roches élastiques et peu perméables. Thèse de D.I.I.P.G. Paris.

\begin{tabular}{|c|c|c|c|c|c|c|c|c|}
\hline Forage & $N^{\circ}$ & Prof. (m) & $\eta^{\circ}$ & $\Delta \eta$ & $\begin{array}{c}\sigma_{n} \\
\text { (bar) }\end{array}$ & $\begin{array}{c}\Delta \sigma_{n} \\
\text { (bar) }\end{array}$ & $\left.\varnothing()^{\circ}\right)$ & $\Delta \varnothing$ \\
\hline A & 3 & 2,7 & 140 & 5 & 14 & $\pm 1,5$ & 0 & 5 \\
A & 4 & 0,90 & 177 & 2 & 6,5 & $\pm 1,5$ & 0 & 5 \\
C & 6 & 0,90 & 152 & 7 & 11 & \pm 3 & 7,5 & 5 \\
C & 7 & 1,50 & 145 & 5 & 11,5 & $\pm 0,5$ & 0 & 5 \\
B & 11 & 2,70 & 140 & 5 & 13 & \pm 1 & 12,5 & 5 \\
\hline
\end{tabular}




\section{ANNEXE}

Le problème des vérins courbes en élasticité plane

Champ de contrainte dans un anneau circulaire

En tout point $\rho, \theta$ de l'anneau, le champ de contrainte aura la forme

$\sigma_{\rho p}=\sum_{-\infty}^{+\infty}(2-2 m) a_{2 m} \rho^{2 m} \cos 2 m \theta-\sum_{-\infty}^{+\infty} a_{2 m}^{\prime} \rho^{2 m} \cos (2 m+2) \theta$

$\sigma_{\theta \theta}=\sum_{-\infty}^{+\infty}(2+2 \mathrm{~m}) \mathrm{a}_{2 \mathrm{~m}} \rho^{2 \mathrm{~m}} \cos 2 \mathrm{~m} \theta+\sum_{-\infty}^{+\infty} \mathrm{a}_{2 \mathrm{~m}}^{\prime} \rho^{2 \mathrm{~m}} \cos (2 \mathrm{~m}+2) \theta$

$\tau_{\rho \theta}=\sum_{-\infty}^{+\infty}(2+2 \mathrm{~m}) \mathrm{a}_{2 \mathrm{~m}} \rho^{2 \mathrm{~m}} \sin 2 \mathrm{~m} \theta+\sum_{-\infty}^{+\infty} \mathrm{a}_{2 m}^{\prime} \rho^{2 \mathrm{~m}} \sin (2 \mathrm{~m}+2) \theta$

où :

$$
a_{2 m}=\frac{P V}{m \pi} \sin .2 m a R_{2}^{-2 m} \lambda^{2} \frac{\lambda^{2 m}\left(1-\lambda^{-4 m+2}\right)-(1+2 m) \lambda^{-2 m}\left(1-\lambda^{2}\right)}{\left(1-4 m^{2}\right)\left(1-\lambda^{2}\right)^{2}-\left(1-\lambda^{4 m+2}\right)\left(1-\lambda^{-4 m+2}\right)}
$$

$a_{2(m-1)}^{\prime}=\frac{P V}{m \pi} \sin .2 m \alpha \lambda^{2} \frac{(1-2 m)\left(\lambda^{2 m+2}-\lambda^{-2 m+2}\right)+\lambda^{-2 m}\left[(1-\lambda)^{4 m+2}-\left(1-4 m^{2}\right)\left(1-\lambda^{2}\right)\right]}{\left(1-4 m^{2}\right)\left(1-\lambda^{2}\right)^{2}-\left(1-\lambda^{4 m+2}\right)\left(1-\lambda^{-4 m+2}\right)}$

$$
x R_{2}^{2-2 m} m= \pm 1, \pm 2, \pm 3
$$

avec $\lambda=R_{1} / R_{2}$

Les valeurs de $a_{0}$ et $a_{-2}^{\prime}$ sont des cas particuliers telles que :

$$
\begin{aligned}
& a_{0}=\frac{P V \alpha}{\pi}\left(\frac{R_{1}^{2}}{R_{1}^{2}-R_{2}^{2}}\right) \\
& a_{-2}^{\prime}=\frac{2 \alpha P V R_{1}^{2} R_{2}^{2}}{\pi\left(R_{1}^{2}-R_{2}^{2}\right)}
\end{aligned}
$$

Cas particulier : Massif infini.

En faisant $R_{2}=\infty$, on retrouve les formules proposées par JAEGER \& COOK (5)

$\sigma r+\sigma \theta \theta=\frac{4 \mathrm{PV}}{\pi} \sum_{m=1}^{\infty} \frac{\sin .2 \mathrm{~m} \alpha}{\mathrm{m}}\left(\frac{\mathrm{R} 1}{\mathrm{P}}\right)^{2 \mathrm{~m}} \cos \cdot 2 \mathrm{~m} \theta$

$\sigma \rho \rho-\sigma \theta \theta=\frac{4 \mathrm{PV} \alpha}{\pi}\left(\frac{\mathrm{R} 1}{\rho}\right)^{2}+\sum_{\mathrm{m}=1}^{\infty} \frac{4 \mathrm{PV}}{\pi} \sin .2 \mathrm{~m} \alpha\left(\frac{\mathrm{R} 1}{\rho}\right)^{2 \mathrm{~m}}\left(1-\frac{\mathrm{R} 1^{2}}{\rho^{2}}\right) \cos 2 \mathrm{~m} \theta$

$\pi \mathrm{r} \theta=\frac{2 \mathrm{PV}}{\pi} \sum_{\mathrm{m}=1}^{\infty}\left(\frac{\mathrm{R} 1}{\rho}\right)^{2 \mathrm{~m}}\left(1-\frac{\mathrm{R} 1^{2}}{\rho^{2}}\right) \sin 2 \mathrm{~m} \alpha \cos 2 \mathrm{~m} \theta$ 


\section{E.N.P.C./D.F.C.A.I. - SESSIONS DE FORMATION - $2^{e}$ TRIMESTRE 1987}

\section{géotechnique, matériaux, structures}

- Nouvelles techniques d'études et de construction sur les sols compressibles

- Techniques de soutènement en élévation

13 au 15 octobre Paris

20 au 23 octobre Paris

Journées d'étude :

- Grands travaux et géologie

- La responsabilité du géotechnicien : pratique professionnelle aspects jurisprudentiels

- Le creusement mécanisé des tunnels

- Compactage
18 et 19 novembre Paris

15 et 16 décembre Paris

15 au 17 décembre Paris 12 au 16 octobre Rouen

programme du premier semestre 1988

Journées d'étude :

- L'évaluation des risques naturels

- La pratique de l'injection et des écrans d'étanchéité

- Stockage en souterrain

- Stabilité des pentes

- Les nouvelles règles parasismiques

- Géotechnique : les enseignements à tirer des accidents survenus aux ouvrages de génie civil

$1^{\text {ex }}$ et 2 mars
8 au 10 mars
22 au 24 mars
26 au 29 avril
$1^{\text {ex }}$ et 2 juin
Journées d'étude
Juin

Paris

Paris

Paris

Grenoble

Grenoble

Paris

Pour toute information, s'adresser à l'E.N.P.C., 28, rue des Saints-Pères, 75007 Paris à Christine ROSE. Tél. 16 (1) 42.60.34.13

\section{GROUND ENGINEERING}

\section{Contents Vol. 20, No. 1, January 1987}

2 Talking Point: Getting physical, by William H. Craig

4 British Geotechnical Society News

5 For the record

7 Geotechnical aspects of the Mexico earthquake - A report on the joint British Geotechnical Society/SECED meeting at the ICE, Westminster, on November 12 , 1986 , by Sarah Springman

9 Geodiary
12 Specification for pile testing (Feration of Piling Specialists)

21 Soil Nailing: Applications and Practice - part 2, by

D.A. Bruce and R.A. Jewell

35 Rawco demonstrates plant for mini-piling

38 Geotechnical Materials: Preventing heave of floor slab of Gatwick's new terminal building; Membrane for lining reservoirs and forming baffles

\section{Contents Vol. 20, No. 2, March 1987}

\author{
2 Talking Point: Value for money, by Ivor Ellis \\ 5 British Geotechnical Society News \\ 5 For the record \\ 8 Current research on foundation design and perfor- \\ mance by the Laboratoires des Ponts et Chaussées, \\ France - Report on a lecture by Dr. Roger Frank at a \\ meeting of the British Geotechnical Society held at the \\ ICE, Westminster, on October 8, 1986, by G. Price \\ 11 Geodiary \\ 15 Piling for Thames-side development
}

\begin{abstract}
21 Observations of the effects of shallow tunnels on buried services, by R.C. Owen

30 Ground Engineering Plant and Equipment: Liebherr Great Britain to market new range of crawler cranes in UK: Akermans to sell Banut piling rig in UK; Complex spraying of concrete for slalom course

31 Underground Services: Stanton design ductile iron linings for Channel Tunnel; Decon to manufacture and
\end{abstract} sell Saltau microtunnelling machines 